Interfaces and Free Boundaries 14 (2012), 167-184

DOI $10.4171 / \mathrm{IFB} / 278$

\title{
Capillary drops on a rough surface
}

\author{
Antoine Mellet \\ Department of Mathematics, Mathematics Building, University of Maryland, College Park, MD \\ 20742-4015, USA \\ E-mail:mellet@math.umd.edu \\ JAMES NOLEN \\ Department of Mathematics, Duke University, Box 90320, Durham, NC 27708, USA \\ E-mail:nolen@math.duke.edu
}

[Received 31 January 2011 and in revised form 17 February 2012]

\begin{abstract}
We study liquid drops lying on a rough planar surface. The drops are minimizers of an energy functional that includes a random adhesion energy. We prove the existence of minimizers and the regularity of the free boundary. When the length scale of the randomly varying surface is small, we show that minimizers are close to spherical caps which are minimizers of an averaged energy functional. In particular, we give an error estimate that is algebraic in the scale parameter and holds with high probability.
\end{abstract}

2010 Mathematics Subject Classification: Primary 76D45; Secondary 35B27, 35R60, 35R35, 49Q10.

Keywords: Capillary drops, homogenization, random media, free boundary problems

\section{Introduction}

\subsection{Liquid drops and Caccioppoli sets}

We consider liquid drops resting on an planar surface with inhomogeneous adhesion properties. A drop is represented by a set $E$ of finite perimeter (also called a Caccioppoli set) contained in $\Omega=\mathbb{R}^{d} \times(0, \infty)$ and having fixed volume $|E|=V>0$. Throughout we will use $x$ to denote a point in $\mathbb{R}^{d}, z$ to denote a point in $[0, \infty)$, and $y=(x, z)$ to denote a point in $\mathbb{R}^{d+1}$. The boundary set $\partial \Omega=\left\{(x, 0): x \in \mathbb{R}^{d}\right\}$ is the solid surface on which the drop is resting.

For a given Caccioppoli set $E \subset \Omega, P(E, \Omega)$ will denote the perimeter of the free surface $\partial E \cap\{z>0\}$ (which represents the liquid-vapor interface) and is defined as follows:

$$
P(E, \Omega)=\sup \left\{\int_{E} \operatorname{div} \mathbf{g}(y) d y: \mathbf{g} \in\left[\mathrm{C}_{0}^{1}(\Omega)\right]^{d+1},|\mathbf{g}| \leqslant 1\right\} .
$$

Because the characteristic function $\phi_{E}$ of a Caccioppoli set is in $\operatorname{BV}(\Omega)$, it has a trace $\bar{\phi}_{E} \in L^{1}(\partial \Omega)$ (see [5]).

We consider a simple situation in which the energy of a drop is the sum of the surface tension energy (proportional to the free surface area) and the wetting energy (resulting from the interactions between the liquid and the solid). After some normalization, we thus assume that the energy of a drop $E$ is given by

$$
\mathscr{J}(E)=P(E, \Omega)-\int_{\mathbb{R}^{d}} \beta(x) \bar{\phi}_{E}(x) d x
$$


where the coefficient $\beta(x) \in \mathbb{R}$ is the relative adhesion coefficient between the liquid and the solid. Positive values $\beta>0$ correspond to a hydrophilic surface, while negative values $\beta<0$ correspond to a hydrophobic surface. It is known that if $\beta(x) \leqslant-1$ for all $x$, then global minimizers of $\mathscr{J}(E)$, under the volume constraint $|E|=V$, are spherical sets in $\Omega$ having no contact with the surface $\partial \Omega$. On the contrary, if $\beta>-1$, absolute minimizers of the functional $\mathscr{J}$ must touch the solid support, though a sphere of volume $V$ is still a local (degenerate) minimizer (see [4, 6]).

In this article we study minimizers of $\mathscr{J}$ when $\beta(x)$ is a random field taking values in the interval $(-1,1)$. Our first result is a proof of the existence and regularity of global minimizers under an additional constraint that confines the drop to a bounded region. We then consider the issue of homogenization. For $\beta(x)=\eta(x / \varepsilon)$ with $\varepsilon>0$ small, we show that global minimizers $E_{\varepsilon}$ are very close to minimizers of a homogenized energy functional $\mathscr{J}_{0}$. In particular, we give an error estimate that is algebraic in $\varepsilon$ as $\varepsilon \rightarrow 0$ and holds with high probability.

\subsection{Free surface, wetted region and contact line}

Given a drop $E$, the free surface of the drop (the liquid-vapor interface) is the set $\partial E \cap\{z>0\}$ whose area (perimeter of $E$ ) is defined above. Note that sets of finite perimeter are defined only up to sets of measure 0 . We will thus normalize $E$ (as in [5]) so that

$$
0<\left|E \cap B_{r}^{+}(y)\right|<\left|B_{r}^{+}(y)\right| \quad \text { for all } y \in \partial E \text { and all } r>0 .
$$

Here and below, $|A|$ denotes the Lebesgue measure of a set $A, B_{r}(y)$ denotes a ball of radius $r$ and center $y$, and $B_{r}^{+}=B_{r} \cap\{z>0\}$.

For a smooth set, the wetted region (liquid-solid interface), is

$$
\Sigma_{E}=\bar{E} \cap\{z=0\},
$$

which is the trace of $E$ on $\partial \Omega$. However in general, it is not obvious that the trace $\bar{\phi}_{E}$ of $\phi_{E}$ is equal to 0 or 1 almost everywhere, so we cannot yet define $\Sigma_{E}$.

Next, the contact line (the liquid-vapor-solid interface) is defined for a smooth set $E$ as the topological boundary of $\Sigma_{E}$ :

$$
C l_{E}=\partial \Sigma_{E}
$$

For a general Caccioppoli set, the contact line $C l_{E}$ is defined as the set of points $x_{0}$ such that $\left(x_{0}, 0\right)$ belongs to $\partial E$, that is such that

$$
\begin{cases}\left|E \cap B_{r}^{+}\left(x_{0}, 0\right)\right|>0 & \text { for all } r>0 \\ \left|(\Omega \backslash E) \cap B_{r}^{+}\left(x_{0}, 0\right)\right|>0 & \text { for all } r>0\end{cases}
$$

We immediately see that if $x_{0} \notin C l_{E}$, then either $\bar{\phi}_{E}$ is zero almost everywhere in a neighborhood of $x_{0}$ or $\bar{\phi}_{E}$ is one almost everywhere in a neighborhood of $x_{0}$. This means that outside of $C l_{E}$, the contact set $\Sigma_{E}$ is well defined.

In Section 2.3, we will show that for minimizers of $\mathscr{J}$, the contact line $C l_{E}$ is indeed a line - it has finite $d-1$ Hausdorff measure. This implies in particular that for minimizers of $\mathscr{J}$, the trace $\bar{\phi}_{E}$ is equal to 0 or 1 almost everywhere, which justifies, a posteriori, our notation $\Sigma_{E}$ for the wetted region ( $\Sigma_{E}$ can indeed be defined as the set of points where $\bar{\phi}_{E}=1$ ). For such sets, we have

$$
\int_{\mathbb{R}^{d}} g(x) \bar{\phi}_{E}(x) d x=\int_{\Sigma_{E}} g(x) d x
$$


for all bounded measurable functions $g$, and

$$
\left|\Sigma_{E}\right|=\int_{\mathbb{R}^{d}} \bar{\phi}_{E}(x) d x .
$$

\subsection{Constant adhesion coefficient}

Before defining the random field $\beta(x)$ and stating our results, we first briefly recall some classical facts about the minimizers of (1.1) when $\beta \equiv \beta^{0} \in(-1,1)$ is constant (see E. Gonzalez et.al. [7] for details and references). For such a homogeneous surface, the corresponding energy functional is

$$
\mathscr{J}_{0}(E)=P(E, \Omega)-\beta^{0} \int_{\mathbb{R}^{d}} \bar{\phi}_{E}(x) d x .
$$

For any given volume $V>0$, we define $\mathscr{E}_{0}(V)=\{E \subset \Omega ; P(E, \Omega)<\infty,|E|=V\}$. It is known that there exists a set $E \in \mathscr{E}_{0}(V)$ which is a minimizer of the energy functional (1.3):

$$
\mathscr{J}(E) \leqslant \mathscr{J}(F), \quad \forall F \in \mathscr{E}_{0}(V) .
$$

Furthermore, Schwarz symmetrization decreases the energy: For every $E \in \mathscr{E}_{0}(V)$, the set

$$
E^{s}=\{(x, z) \in \Omega ;|x|<\rho(z)\}, \quad \text { where } \rho(z)=\left(\omega_{d}^{-1} \int_{\mathbb{R}^{d}} \phi_{E}(x, z) d x\right)^{\frac{1}{d}}
$$

is a Caccioppoli set with the same volume $V$, and we have the inequality

$$
\mathscr{J}_{0}\left(E^{s}\right) \leqslant \mathscr{J}_{0}(E) .
$$

The constant $\omega_{d}$ is the measure of the unit ball in $\mathbb{R}^{d}$. Equality holds in (1.4) if and only if $E$ is symmetric (i.e. $E^{s}=E$ ). This fact implies that any minimizer should have axial symmetry, and it can be shown that minimizers are spherical caps, which are the intersection of the upper-half space $\Omega$ with a ball $B_{\rho_{0}}\left(x_{0}, z_{0}\right) \in \mathbb{R}^{d+1}$ having radius $\rho_{0}$ and center $\left(x_{0}, z_{0}\right)$. We use

$$
B_{\rho_{0}}^{+}\left(x_{0}, z_{0}\right)=B_{\rho_{0}}\left(x_{0}, z_{0}\right) \cap\{z>0\}
$$

to denote such a spherical cap. In this case the wetted region $\Sigma_{E}$ is a disc of radius $\sqrt{\rho_{0}^{2}-z_{0}^{2}}$, where $z_{0}, \rho_{0}$ are such that the Young-Laplace law and volume constraint are satisfied:

$$
\frac{z_{0}}{\rho_{0}}=\beta^{0}, \quad V=\omega_{d} \int_{-z_{0}}^{\rho_{0}}\left(\rho_{0}^{2}-r^{2}\right)^{d / 2} d r
$$

Finally, we recall the following stability result for the minimization problem with constant adhesion coefficient (see [1]), which will play an important role in our analysis for the case of heterogeneous adhesion coefficient:

THEOREM 1.1 Suppose a set $E \in \mathscr{E}_{0}(V)$ and $R>0$ is such that

$E$ is contained in a ball $B_{R} \subset \Omega$, 
and

$$
\exists \delta>0 \quad \text { s.t. } \mathscr{J}_{0}(E) \leqslant \mathscr{J}_{0}(F)+\delta, \quad \forall F \in \mathscr{E}_{0}(V) .
$$

There exists a universal $s>0$, a constant $C$ (depending on $R$ ), and a point $x_{0} \in \mathbb{R}^{d}$ such that

$$
\left|E \triangle B_{\rho_{0}}^{+}\left(x_{0}, z_{0}\right)\right| \leqslant C \delta^{s}
$$

where $\rho_{0}$ and $z_{0}$ satisfy (1.5).

The notation $E \triangle B$ denotes the symmetric difference of sets $E, B$. If $E$ also satisfies some nondegeneracy conditions, then Theorem 1.1 implies the uniform stability in the following sense: For any $\eta>0$, there exists $\delta_{0}$ such that if (1.7) holds with $\delta<\delta_{0}$, then

$$
B_{(1-\eta) \rho_{0}}^{+}\left(x_{0}, z_{0}\right) \subset E \subset B_{(1+\eta) \rho_{0}}^{+}\left(x_{0}, z_{0}\right) .
$$

In other words, the free surface $\partial E \cap\{z>0\}$ is between $\partial B_{(1+\eta) \rho_{0}}^{+}\left(x_{0}, z_{0}\right)$ and $\partial B_{(1-\eta) \rho_{0}}^{+}\left(x_{0}, z_{0}\right)$.

\subsection{Random adhesion coefficient}

We now describe the framework of this paper and state our main result:

Let $\left\{\beta_{k}\right\}_{k \in \mathbb{Z}^{d}}$ be a collection of independent, identically distributed random variables satisfying

$$
\mathbb{P}\left(\beta_{k} \in\left(\beta_{\text {min }}, \beta_{\text {max }}\right)\right)=1, \quad \mathbb{E}\left[\beta_{k}\right]=\beta^{0},
$$

for some constants $-1<\beta_{\min } \leqslant \beta_{\max }<1$. We use $(H, \mathcal{F}, \mathbb{P})$ to refer to the probability space over which these random variables are defined. For $\varepsilon>0$ and $k \in \mathbb{Z}^{d}$, let $Q_{k}^{\varepsilon}=\varepsilon k+[0, \varepsilon)^{d}$ denote the nonoverlapping cubes of size $\varepsilon$ with corners at the points $\varepsilon \mathbb{Z}^{d}$. Then, we define the random adhesion coefficient $\beta^{\varepsilon}(x)$ according to

$$
\beta^{\varepsilon}(x)=\beta_{k}, \quad \text { if } x \in Q_{k}^{\varepsilon} .
$$

Observe that $\beta^{\varepsilon}(x)=\beta^{1}(x / \varepsilon)$. We will use $\mathscr{J}_{\varepsilon}$ and $\mathscr{J}_{0}$ to denote the energy functionals associated with $\beta^{\varepsilon}(x)$ and $\beta^{0}$, respectively, replacing $\beta(x)$ in (1.1).

In full generality, the existence of a minimizer for (1.1) can be delicate to establish. Indeed, it easy to construct a function $\beta(x)$ for which minimizing sequences do not converge in $L^{1}$. For example, let $\beta(x)$ be a strictly increasing function of $x_{1}$ (such as $\beta(x)=\frac{1}{\pi} \arctan \left(x_{1}\right)$, which satisfies $\left.\lim _{x_{1} \rightarrow \pm \infty} \beta(x)= \pm \frac{1}{2} \in(-1,1)\right)$. In that case, any minimizing sequence will drift toward $x_{1} \rightarrow+\infty$ and will not converge in $L^{1}$.

In order to avoid such behavior, we will assume that the drops must stay inside a "container" of the form

$$
U=B_{R} \times[0, \infty)
$$

where $B_{R}$ is a ball of radius $R$ in $\mathbb{R}^{d}$ ( $R$ will always be assumed to be large enough, so that a ball of volume $V$ fits in $U$ ). The "bottom" of the container is thus $B_{R} \times\{0\}$, while the "wall" is $\partial B_{R} \times[0, \infty)$. We will assume that the wall of the container is completely hydrophobic. One way to take that into account is to include the area of $E \cap\left(\partial B_{R} \times[0, \infty)\right)$ in the wetting energy, with $\beta=-1$. Another way is to include the area $E \cap\left(\partial B_{R} \times[0, \infty)\right)$ in the free surface area. Choosing the later approach, we define

$$
\mathscr{E}(V)=\{E \subset U: P(E, \Omega)<\infty, \quad|E|=V\},
$$


and for all $E \in \mathscr{E}(V)$ we define the energy

$$
\mathscr{J}_{\varepsilon}(E)=P(E, \Omega)-\int_{\mathbb{R}^{d}} \beta^{\varepsilon}(x) \bar{\phi}_{E}(x) d x .
$$

Our main result is:

THEOREM 1.2 With probability one and for all $\varepsilon>0$, there exists a Caccioppoli set $E_{\varepsilon} \in \mathscr{E}(V)$ such that

$$
\mathscr{J}_{\varepsilon}\left(E_{\varepsilon}\right)=\min _{E \in \mathscr{E}(V)} \mathscr{J}_{\varepsilon}(E)
$$

Furthermore, for any $r>(d+1) / d$ there are constants $K_{r}, K_{2}$, and $K_{3}$ such that if $\alpha>0$ and $\varepsilon<K_{r} \min \left(1, \alpha^{r}\right)$, then there is a random point $x_{0} \in \mathbb{R}^{d}$ such that

$$
\left|E_{\varepsilon} \triangle B_{\rho_{0}}^{+}\left(x_{0}, z_{0}\right)\right| \leqslant C \alpha^{s}
$$

holds, except possibly on a $\mathcal{F}$-measurable set of measure less than $K_{2} e^{-K_{3} \varepsilon^{-d} \alpha^{2}}$. The constants $K_{2}$ and $K_{3}$ depend on $V$, but not on $r$.

The exponent $s>0$ and the constant $C$ are the same as those appearing in Theorem 1.1. The estimate (1.12) shows that when $\varepsilon$ is small, minimizers of $\mathscr{J}_{\varepsilon}$ are close to a spherical cap which minimizes $\mathscr{J}_{0}$, the energy functional associated with the homogeneous medium $\beta \equiv \beta^{0}$. It is not clear whether the random variable $\left|E_{\varepsilon} \Delta B_{\rho_{0}}^{+}\left(x_{0}, z_{0}\right)\right|$ is $\mathcal{F}$-measurable; we do not claim that $x_{0}$ is $\mathcal{F}$-measurable. Nevertheless, the set where (1.12) may not hold must be contained in a $\mathcal{F}$ measurable set of measure less than $K_{2} e^{-K_{3} \varepsilon^{-d} \alpha^{2}}$. Therefore, if we choose $0<p<d /(d+1)$, then the estimate (1.12) says that $\left|E_{\varepsilon} \triangle B_{\rho_{0}}^{+}\left(x_{0}, z_{0}\right)\right| \leqslant C \varepsilon^{s p}$ with very high probability as $\varepsilon \rightarrow 0$. In particular, if the surface dimension is $d=2$, then the error $\left|E_{\varepsilon} \triangle B_{\rho_{0}}^{+}\left(x_{0}, z_{0}\right)\right|$ is almost $O\left(\varepsilon^{s 2 / 3}\right)$.

Theorem 1.2 builds upon work of Caffarelli and Mellet [1] where they studied the case of periodic adhesion coefficient $\beta(x / \epsilon)$. In that setting, they proved the existence of minimizers which are close to a spherical cap when $\epsilon$ is small. For a random adhesion coefficient, however, some technical issues arise related to the construction of minimizers, as we have mentioned. In Section 2, we prove the existence and regularity properties of minimizers of $\mathscr{J}$ with the additional constraint $E \subset U$. We prove the error estimate (1.12) later in Section 3. In view of Theorem 1.1, the error estimate follows from a suitable bound on the energy difference $\mathscr{J}_{\varepsilon}\left(E_{\varepsilon}\right)-\mathscr{J}_{0}\left(E_{\varepsilon}\right)$. To obtain such a bound, we make use of the uniform regularity of the free boundary and apply concentration inequalities for sums of independent random variables. We refer to [3] for another example of an error estimate for a nonlinear, stochastic homogenization problem.

In [2], Caffarelli and Mellet used the result of [1] to show that homogenization leads to hysteresis phenomena, in the sense that there exist local minimizers for $\varepsilon>0$ that converge to non-spherical drops (with contact angle larger than the average of $\beta$ on parts of the contact line). Such a result could be extended to the random framework, but would require strong assumptions on the structure of the oscillations of $\beta$. Several results have also been obtained concerning the homogenization of dynamic contact angle conditions (moving liquid drops). In particular, Kim in [9, 10] has studied the periodic homogenization of some Hele-Shaw type models for dynamic contact angle, and established error estimates [8]. Finally, the random homogenization for the Hele-Shaw free boundary problem is studied in [11].

Acknowledgment. AM was partially supported by NSF grant DMS-0901340. JN was partially supported by NSF grant DMS-1007572. 


\section{Existence and regularity of minimizers}

In this section, we consider the functional $\mathscr{J}$ defined by (1.1) with $\beta(x)$ being any measurable function that satisfies

$$
-1<\beta_{\min } \leqslant \beta(x) \leqslant \beta_{\max }<1, \quad \text { for all } x \in \mathbb{R}^{d} .
$$

Our goal is to study the existence and regularity of minimizers for $\mathscr{J}$ (with the volume constraint). Similar results were proved in [1] in the periodic framework, and can be generalized. However, in the periodic case, it is not necessary to constrain $E$ to a bounded set $U$ : the periodicity prevents minimizing sequences from drifting away. As pointed out in the introduction, in the general case, we need to work within a bounded set $U$ in order to find a minimizer, and we have to check carefully that this additional constraint does not cause any problems.

We will show:

Proposition 2.1 For any measurable function $\beta(x)$ satisfying (2.1), there exists $E \in \mathscr{E}(V)$ such that

$$
\mathscr{J}(E)=\min _{F \in \mathscr{E}(V)} \mathscr{J}(F)
$$

The contact line $C l_{E}$ (defined by (1.2)) has finite $(d-1)$-Hausdorff measure and there exists a set $\Sigma_{E}$ such that

$$
\int_{\mathbb{R}^{d}} g(x) \bar{\phi}_{E}(x) d x=\int_{\Sigma_{E}} g(x) d x, \quad \text { for all } g \in \mathrm{e}_{0}^{1}\left(\mathbb{R}^{d}\right) .
$$

Finally, there exists a universal constant $C>0$ such that

$$
C^{-1} V^{\frac{d}{d+1}} \leqslant P(E, \Omega) \leqslant C V^{\frac{d}{d+1}}, \quad\left|\Sigma_{E}\right| \leqslant C V^{\frac{d}{d+1}}
$$

and

$$
\mathcal{H}^{d-1}\left(C l_{E}\right) \leqslant C V^{\frac{d-1}{d+1}} .
$$

\section{$2.1 \quad$ Existence of a minimizer}

Because subsets of $B V(\Omega)$ are pre-compact in $L_{l o c}^{1}(\Omega)$, Lemma 2.1 and 2.2 below will yield the existence of minimizers provided that we stay within a bounded subset of $\mathbb{R}^{d+1}$. We thus define

$$
U_{T}=B_{R} \times[0, T]
$$

and

$$
\mathscr{E}_{T}(V)=\left\{E \in \mathscr{E}(V) ; E \subset U_{T}\right\},
$$

and we assume that $T$ is large enough, so that $\mathscr{E}_{T}(V)$ is not empty. We then have the following proposition:

PROPOSITION 2.2 There exists $E \in \mathscr{E}_{T}(V)$ satisfying

$$
\mathscr{J}(E)=\min _{F \in \mathscr{E}_{T}(V)} \mathscr{J}(F) .
$$

Moreover, there exists a universal constant $C$ such that

$$
C^{-1} V^{\frac{d}{d+1}} \leqslant P(E, \Omega) \leqslant C V^{\frac{d}{d+1}} \quad \text { and } \quad \int_{\mathbb{R}^{d}} \bar{\phi}_{E}(x) d x \leqslant C V^{\frac{d}{d+1}} .
$$


Proposition 2.2 follows from the following lemmas:

LEMma 2.1 The functional $\mathscr{J}$ is lower semicontinuous with respect to the $L^{1}$ topology: If $\left\{E_{j}\right\}_{j=1}^{\infty}$ is a sequence of Caccioppoli sets such that $\phi_{E_{j}} \longrightarrow \phi_{E}$ in $L^{1}(\Omega)$ then

$$
\mathscr{J}(E) \leqslant \liminf _{j \rightarrow \infty} \mathscr{J}\left(E_{j}\right)
$$

LEMMA 2.2 If $\beta$ satisfies (2.1), then

$$
\mathscr{J}(E) \geqslant \frac{1-\beta_{\max }}{2} P(E, \Omega)+\frac{1-\beta_{\max }}{2} \int_{\mathbb{R}^{d}} \bar{\phi}_{E}(x) d x
$$

for all $E \in \mathscr{E}(V)$.

Proof of Lemma 2.2. We recall that for all $\mathbf{h} \in\left[\mathrm{C}^{1}\left(\mathbb{R}^{d+1}\right)\right]^{d+1}$, we have

$$
\int_{\Omega} \phi_{E} \operatorname{div} \mathbf{h}=-\int_{\Omega}\left\langle\mathbf{h}, D \phi_{E}\right\rangle+\int_{\mathbb{R}^{d}} \bar{\phi}_{E}(x) \mathbf{h}_{d+1}(x, 0) d x .
$$

If $g(x)$ is a non-negative measurable function, then taking $\mathbf{h}=(0, \ldots, 0, g(x))$, we deduce:

$$
\int_{\Omega} g(x)\left|D \phi_{E}\right| d y \geqslant \int_{\mathbb{R}^{d}} g(x) \bar{\phi}_{E} d x .
$$

Hence

and therefore

$$
\int_{\Omega} \frac{1+\beta(x)}{2}\left|D \phi_{E}\right| d y \geqslant \int_{\mathbb{R}^{d}} \frac{1+\beta(x)}{2} \bar{\phi}_{E}(x) d x
$$

$$
\int_{\Omega}\left(1-\frac{1-\beta(x)}{2}\right)\left|D \phi_{E}\right| d y \geqslant \int_{\mathbb{R}^{d}}\left(\frac{1-\beta(x)}{2}+\beta(x)\right) \bar{\phi}_{E}(x) d x
$$

which gives

$$
\mathscr{J}(E) \geqslant \int_{\Omega} \frac{1-\beta(x)}{2}\left|D \phi_{E}\right| d y+\int_{\mathbb{R}^{d}} \frac{1-\beta(x)}{2} \bar{\phi}_{E}(x) d x
$$

and implies Lemma 2.2.

Proof of Proposition 2.2. Consider a minimizing sequence $\phi_{E_{j}}$ with $E_{j} \in \mathscr{E}_{T}(V)$. Lemma 2.2 and the fact that $U_{T}$ is bounded imply that $\left(\phi_{E_{j}}\right)_{j}$ is bounded in $B V\left(U_{T}\right)$ and thus compact in $L^{1}\left(U_{T}\right)$. Hence there exists $E$ such that $\phi_{E_{j}} \longrightarrow \phi_{E}$. Using Lemma 2.1 we deduce $E$ is a minimizer of $\mathscr{J}$ in $\mathscr{E}_{T}(V)$.

Next, we remark that (2.8) implies (with $g=1$ )

$$
P(E, \Omega) \geqslant \int_{\mathbb{R}^{d}} \bar{\phi}_{E}(x) d x .
$$

We recall the isoperimetric inequality

$$
|E|^{\frac{d}{d+1}}=V^{\frac{d}{d+1}} \leqslant \mu_{d+1} P\left(E, \mathbb{R}^{d+1}\right)
$$


and (2.7) gives for all $\mathbf{h} \in\left[\mathrm{C}_{0}^{1}\left(\mathbb{R}^{d+1}\right)\right]^{d+1}$ with $|\mathbf{h}| \leqslant 1$

$$
\begin{aligned}
\left|\int_{\mathbb{R}^{d+1}} \phi_{E} \operatorname{div} \mathbf{h}\right| & \leqslant \int_{\Omega}\left|D \phi_{E}\right|+\int_{\mathbb{R}^{d}} \bar{\phi}_{E}(x) d x \\
& \leqslant P(E, \Omega)+\int_{\mathbb{R}^{d}} \bar{\phi}_{E}(x) d x .
\end{aligned}
$$

Taking the supremum over all such $h$, we deduce

$$
P\left(E, \mathbb{R}^{d+1}\right) \leqslant P(E, \Omega)+\int_{\mathbb{R}^{d}} \bar{\phi}_{E}(x) d x
$$

and therefore

$$
V^{\frac{d}{d+1}} \leqslant \mu_{d+1}\left(P(E, \Omega)+\int_{\mathbb{R}^{d}} \bar{\phi}_{E}(x) d x\right) .
$$

Using (2.9), we deduce

$$
V^{\frac{d}{d+1}} \leqslant 2 \mu_{d+1} P(E, \Omega)
$$

Moreover, we have

$$
\mathscr{J}(E) \geqslant P(E, \Omega)-\beta_{\max } \int_{\mathbb{R}^{d}} \bar{\phi}_{E}(x) d x \geqslant\left(1-\beta_{\max }\right) P(E, \Omega) .
$$

and since $\mathscr{J}(E) \leqslant \mathscr{J}(B)$ where $B \subset \Omega$ is a ball with volume $V$, we also have

$$
\left(1-\beta_{\max }\right) P(E, \Omega) \leqslant \mathscr{J}(B)=\mu_{d+1}^{-1} V^{\frac{d}{d+1}} .
$$

Combining (2.9), (2.11) and (2.12), we get (2.6).

In order to prove the first part of Proposition 2.1, it remains to prove that for $T$ large enough, the minimizer for $\mathscr{J}$ in $\mathscr{E}_{T}(V)$ provided by Proposition 2.2 is actually a minimizer in $\mathscr{E}(V)$. This follows from the following result, the proof of which can be found in [1]:

LEMmA 2.3 There exists $T_{1}$ such that for all $T \geqslant T_{1}$, there exists a minimizer $E$ of $\mathscr{J}$ in $\mathscr{E}_{T}$ that satisfies

$$
E \in \mathscr{E}_{T_{1}}(V)
$$

\subsection{Non-degeneracy estimates and weak regularity of the contact line}

In this section, we prove the Hausdorff estimate (2.4). First, we need to show that minimizers of $\mathscr{J}$ enjoy some non-degeneracy properties which will also be useful later on. Throughout this section, $E$ denotes a minimizer of $\mathscr{J}$ in $\mathscr{E}(V)$, as constructed in the previous section.

We recall the following simple fact:

$$
\min _{\mathscr{E}(V)} \mathscr{J} \leqslant \min _{\mathscr{E}(V+d V)} \mathscr{J} \leqslant \min _{\mathscr{E}(V)} \mathscr{J}+C V^{-\frac{1}{d+1}} d V .
$$

The first inequality can be obtained simply taking the minimizer with volume $V+d V$ and chopping a piece of volume $d V$ at the top. For the second inequality, we consider the minimizer $E$ with volume $V$ and take a vertical dilation $\left.E_{t}=\left\{(x, z) ;\left(x,(1+t)^{-1} z\right) \in E\right)\right\}$. Then, for $t=d V / V$, $E_{t}$ is an admissible set of volume $V+d V$. See [1] for details.

We now prove the following non-degeneracy estimate: 
LEMMA 2.4 Let $\left(x_{0}, z_{0}\right) \in \partial E$ with $z_{0}>0$. There exists $c$, universal constant, such that for all $r \leqslant z_{0}$ we have

$$
\begin{aligned}
& \left|B_{r}\left(x_{0}, z_{0}\right) \cap E\right| \geqslant c r^{d+1}, \\
& \left|B_{r}\left(x_{0}, z_{0}\right) \backslash E\right| \geqslant c r^{d+1} .
\end{aligned}
$$

Proof. In order to prove the first inequality, we define (for $r \leqslant z_{0}$ ):

$$
U(r)=\left|B_{r}\left(x_{0}, z_{0}\right) \cap E\right|, \quad S(r)=\mathscr{H}^{n}\left(\partial B_{r}\left(x_{0}, z_{0}\right) \cap E\right)
$$

and

$$
A=P\left(E, B_{r}\left(x_{0}, z_{0}\right)\right) .
$$

Then, using (2.13) and the fact that $E$ is a minimizer, we have

$$
\mathscr{J}\left(E \backslash B_{r}\right)=\mathscr{J}(E)-A+S \geqslant \min _{F \in \mathscr{E}(V-U)} \mathscr{J}(F) \geqslant \mathscr{J}(E)-C U
$$

and therefore

$$
A \leqslant S(r)+C U(r) .
$$

The isoperimetric formula then yields

$$
U(r)^{\frac{d}{d+1}} \leqslant 2 \mu_{d+1}(S(r)+C U(r)) .
$$

Noticing that $U^{\prime}(r)=S(r)$, and using the fact that $U(0)=0$ and $U(r)>0$ for all $r>0$, Gronwall's Lemma gives the first inequality.

The second inequality is proved in a similar way, by estimating $\mathscr{J}\left(E \cup B_{r}\right)$. However, we have to be a little bit careful here, since $B_{r}$ might not lie entirely in the domain $U$. But defining $d_{0}=d\left(x_{0}, \partial U\right)$ the distance of $x_{0}$ from the boundary of $U$ (note that we can have $d_{0}=0$ ), we can still prove

$$
\left|B_{r}\left(x_{0}, z_{0}\right) \backslash E\right| \geqslant c r^{d+1}, \quad \text { for all } r \leqslant d_{0} .
$$

For $r>d_{0}$, we then have (since $E \subset U$ )

$$
\begin{aligned}
\left|B_{r}\left(x_{0}, z_{0}\right) \backslash E\right| & \geqslant\left|B_{d_{0}}\left(x_{0}, z_{0}\right) \backslash E\right|+\left|B_{r}\left(x_{0}, z_{0}\right) \backslash u\right| \\
& \geqslant c d_{0}^{d+1}+\left|B_{r}\left(x_{0}, z_{0}\right) \backslash u\right| .
\end{aligned}
$$

Finally, we note that

$$
\left|B_{r}\left(x_{0}, z_{0}\right) \backslash u\right| \geqslant \alpha_{1} r^{d+1}-\alpha_{2} d_{0}^{d+1}
$$

and so replacing $\left|B_{r}\left(x_{0}, z_{0}\right) \backslash U\right|$ by $\gamma\left|B_{r}\left(x_{0}, z_{0}\right) \backslash U\right|$ for some small enough $\gamma$ in the previous inequality we deduce

$$
\left|B_{r}\left(x_{0}, z_{0}\right) \backslash E\right| \geqslant c r^{d+1}, \quad \text { for all } r<z_{0} .
$$

Next, we want to derive similar non-degeneracy estimate in the neighborhood of the contact line: 
LEMMA 2.5 If $x_{0} \in C l_{E}$, then

$$
\left|E \cap B_{r}^{+}\left(x_{0}, 0\right)\right| \geqslant c r^{d+1}
$$

and

$$
\left|(\Omega \backslash E) \cap B_{r}^{+}\left(x_{0}, 0\right)\right| \geqslant c r^{d+1}
$$

for every $r$ such that $\left|E \cap B_{r}^{+}\left(x_{0}, 0\right)\right| \leqslant c_{1} V$.

Proof. For $r>0$, we define

$$
U(r)=\left|B_{r}^{+}\left(x_{0}, 0\right) \cap E\right|, \quad \Sigma(r)=\int_{B_{r}\left(x_{0}\right)} \bar{\phi}_{E}(x) d x,
$$

and

$$
S(r)=\mathscr{H}^{n}\left(\partial B_{r}^{+}\left(x_{0}, 0\right) \cap E\right), \quad A=P\left(E, B_{r}^{+}\left(x_{0}, 0\right)\right)
$$

(note that for a smooth enough set, we have $\Sigma(r)=\left|B_{r}\left(x_{0}\right) \cap \Sigma_{E}\right|$ ).

We then have

$$
\mathscr{J}\left(E \backslash B_{R}^{+}\left(x_{0}, 0\right)\right) \leqslant \mathscr{J}(E)-A+\beta_{\max } \Sigma+S,
$$

and since

$$
\mathscr{J}\left(E \backslash B_{R}^{+}\left(x_{0}, 0\right)\right) \geqslant \mathscr{J}(E)-C U,
$$

we deduce

$$
A \leqslant \beta_{\max } \Sigma+S+C U .
$$

If $E^{\prime}=E \cap B_{r}^{+}\left(x_{0}, 0\right)$, then the equality

$$
\int_{\Omega} \phi_{E^{\prime}} \operatorname{div} \mathbf{h}=-\int_{\Omega}\left\langle\mathbf{h}, D \phi_{E^{\prime}}\right\rangle+\int_{\mathbb{R}^{d}} \bar{\phi}_{E^{\prime}}(x) \mathbf{h}_{d+1}(x, 0) d x
$$

with $\mathbf{h}(y)=(0, \ldots, 0,1)$ implies that

$$
\Sigma \leqslant S+A
$$

Inequalities (2.15) and (2.17) imply

$$
\left(1-\beta_{\max }\right) \Sigma \leqslant 2 S+C U
$$

and

$$
A \leqslant C S+C U
$$

Finally, the isoperimetric inequality (as in (2.10)) yields

$$
\begin{aligned}
U^{\frac{d}{d+1}} & \leqslant \mu_{d+1}(\Sigma+S+A) \\
& \leqslant C S+C U .
\end{aligned}
$$

Using the fact that $S=U^{\prime}$, Gronwall lemma and (1.2) give the first inequality in Lemma 2.5.

The second inequality follows in a similar fashion.

We deduce: 
COROLlary 2.1 If $x_{0} \in C l_{E}$, then

$$
P\left(E, B_{r}^{+}\left(x_{0}, 0\right)\right) \geqslant c r^{d}
$$

for every $r$ such that $\left|E \cap B_{r}^{+}\left(x_{0}, 0\right)\right| \leqslant c_{1} V$.

Proof of Corollary 2.1. Let

$$
\begin{array}{ll}
V_{1}(r)=\left|E \cap B_{r}^{+}\left(x_{0}, 0\right)\right|, & S_{1}(r)=\mathscr{H}^{n}\left(E \cap \partial B_{r}^{+}\left(x_{0}, 0\right)\right), \\
V_{2}(r)=\left|(\Omega \backslash E) \cap B_{r}^{+}\left(x_{0}, 0\right)\right|, & S_{2}(r)=\mathscr{H}^{n}\left((\Omega \backslash E) \cap \partial B_{r}^{+}\left(x_{0}, 0\right)\right) .
\end{array}
$$

Lemma 2.5 yields

$$
V_{i}(r) \geqslant c r^{d+1} \quad i=1,2 .
$$

Moreover, the isoperimetric inequality gives (after reflecting the set $E$ about $z=0$ )

$$
\begin{aligned}
& \left(2 V_{1}\right)^{\frac{d}{d+1}} \leqslant \mu_{d+1}\left(2 S_{1}+2 P\left(E, B_{r}^{+}\left(x_{0}, 0\right)\right)\right), \\
& \left(2 V_{2}\right)^{\frac{d}{d+1}} \leqslant \mu_{d+1}\left(2 S_{2}+2 P\left(E, B_{r}^{+}\left(x_{0}, 0\right)\right)\right)
\end{aligned}
$$

and (since we have equality in the isoperimetric inequality for the ball)

$$
\left(2\left(V_{1}+V_{2}\right)\right)^{\frac{d}{d+1}}=\mu_{d+1} 2\left(S_{1}+S_{2}\right) .
$$

It follows that

$$
V_{1}^{\frac{d}{d+1}}+V_{2}^{\frac{d}{d+1}}-\left(V_{1}+V_{2}\right)^{\frac{d}{d+1}} \leqslant 4 \mu_{d+1} P\left(E, B_{r}^{+}\left(x_{0}, 0\right)\right),
$$

which yields the result thanks to (2.20).

\subsection{Hausdorff dimension of the contact line}

The nondegeneracy estimates enable us to prove the following partial regularity result for the contact line, and complete the proof of Proposition 2.1:

Proposition 2.3 Let $E \in \mathscr{E}(V)$ minimize $\mathscr{J}$. Then, the contact line $C l_{E}$ in $\mathbb{R}^{d}$ has finite $(d-1)$ Haussdorff measure, and there exists a universal constant $C$ such that

$$
\mathcal{H}^{d-1}\left(C l_{E}\right) \leqslant C V^{\frac{d-1}{d+1}} .
$$

In particular, the function $\bar{\phi}_{E}$ is equal to 0 or 1 almost everywhere, and we can define the wetted region $\Sigma_{E} \subset \mathbb{R}^{d}$ as the set where $\bar{\phi}_{E}=1$.

This proposition is a consequence of Corollary 2.1 and the following lemma.

Lemma 2.6 There exists a universal constant $C$ such that

$$
P(E,\{0<z<t\}) \leqslant C V^{\frac{d-1}{d+1}} t .
$$


Proof. We cut from $E$ all the points for which $z \leqslant t$ and lower it by $t$. This defines

$$
F=\{(x, z) ;(x, z+t) \in E\} \cap\{z>0\} .
$$

Then we have $|F| \leqslant|E|-C t$ and so by (2.13) we have $\mathscr{J}(E) \leqslant \mathscr{J}(F)+C t$. Moreover

$$
\mathscr{J}(E)-\mathscr{J}(F)=P(E,\{0<z<t\})-\int \beta(x / \varepsilon)\left[\phi_{E}(x, 0)-\phi_{E}(x, t)\right] d x,
$$

(where $\phi_{E}(x, 0)$ and $\phi_{E}(x, t)$ denotes the trace of $\phi_{E}$ on $\{z=0\}$ and $\{z=t\}$ ) but if $x$ belongs to the symmetric difference of $E \cap\{z=0\}$ and $E \cap\{z=t\}$, then, going from the slice $\{z=0\}$ to the slice $\{z=t\}$, we must cross $\partial E$, and therefore

$$
\int\left|\phi_{E}(x, 0)-\phi_{E}(x, t)\right| d x \leqslant P(E,\{0<z<t\}) .
$$

We deduce

$$
\left(1-\beta_{\max }\right) P(E,\{0<z<t\}) \leqslant C V^{\frac{d-1}{d+1}} t
$$

which completes the proof.

Proof of Proposition 2.3. Let $\cup_{j \in J} B_{\delta}\left(x_{j}\right)$ be a covering of $C l_{E}$ by balls of radius $\delta$ with finite overlapping. Then by Corollary 2.1, we have $P\left(E, B_{\delta}^{+}\left(x_{j}\right)\right) \geqslant C \delta^{d}$. Due to the finite overlapping property,

$$
\sum_{j \in J} P\left(E, B_{\delta}^{+}\left(x_{j}\right)\right) \leqslant C P(E,\{0<z<\delta\}) \leqslant C V^{\frac{d-1}{d+1}} \delta .
$$

Therefore, the number of balls is less than $C V^{\frac{d-1}{d+1}} \delta^{1-d}$. Hence the stated result.

\section{Proof of Theorem 1.2}

The first part of Theorem 1.2 follows immediately from Proposition 2.1: with probability one $\beta(x)=\beta^{\varepsilon}(x)$ satisfies the hypotheses of Proposition 2.1 for all $\varepsilon>0$. So, for all $\varepsilon>0$ there exists a minimizer $E_{\varepsilon} \in \mathscr{E}(V)$ of $\mathscr{J}_{\varepsilon}$ and by Proposition 2.3, we can define the wetted region $\Sigma_{\varepsilon}=E_{\varepsilon} \cap\{z=0\}$ and the contact line $\partial \Sigma_{\varepsilon}=C l_{E_{\varepsilon}}$.

The minimizer $E_{\varepsilon}$ is a random set in $\mathbb{R}^{d}$. Nevertheless, we have shown that there is a deterministic constant $C$, independent of $\varepsilon$, such that $E_{\varepsilon}$ satisfies:

$$
P\left(E_{\varepsilon}, \Omega\right) \leqslant C V^{\frac{d}{d+1}}, \quad\left|\Sigma_{\varepsilon}\right| \leqslant C V^{\frac{d}{d+1}}, \quad \text { and } \quad \mathcal{H}^{d-1}\left(\partial \Sigma_{\varepsilon}\right) \leqslant C V^{\frac{d-1}{d+1}} .
$$

We recall that $\mathscr{J}_{0}$ denotes the functional defined as $\mathscr{J}_{\varepsilon}$ but with the constant $\beta^{0}$ in the place of $\beta^{\varepsilon}(x)$, and we denote by $E_{0}$ its minimizer with volume constraint $\left|E_{0}\right|=V$. This set is a spherical cap $E_{0}=B_{\rho}^{+}\left(x_{0}, z_{0}\right)$, and it is unique up to translation in $x_{0}$. The wetted region is $\Sigma_{0}=\Sigma_{E_{0}}$. In order to estimate the symmetric difference $\left|E_{\varepsilon} \triangle E_{0}\right|$, we will apply Theorem 1.1. To this end, we will show that for any $\alpha>0$, the set $E_{\varepsilon}$ satisfies

$$
\mathscr{J}_{0}\left(E_{\varepsilon}\right) \leqslant \mathscr{J}_{0}\left(E_{0}\right)+\alpha
$$

with high probability if $\varepsilon$ is sufficiently small. More precisely, we will show 
THEOREM 3.1 Let $r>(d+1) / d$. There are constants $K_{r}, K_{2}, K_{3}$ such that if $\alpha>0$ and $\varepsilon \leqslant$ $K_{r} \min \left(1, \alpha^{r}\right)$, the minimizer $E_{\varepsilon}$ of $\mathscr{J}_{\varepsilon}$ satisfies

$$
\mathbb{P}\left(\mathscr{J}_{0}\left(E_{\varepsilon}\right) \geqslant \mathscr{J}_{0}\left(E_{0}\right)+\alpha\right) \leqslant K_{2} e^{-K_{3} \varepsilon^{-d} \alpha^{2}} .
$$

The bound (1.12) now follows immediately from this estimate and Theorem 1.1.

Proof of Theorem 3.1. By definition of $\mathscr{J}_{0}, \mathscr{J}_{\varepsilon}$, and $E_{\varepsilon}$, we have:

$$
\begin{aligned}
\mathscr{J}_{0}\left(E_{\varepsilon}\right) & =\mathscr{J}_{\varepsilon}\left(E_{\varepsilon}\right)+\int_{\Sigma_{\varepsilon}}\left(\beta^{\varepsilon}(x)-\beta^{0}\right) d x \\
& \leqslant \mathscr{J}_{\varepsilon}\left(E_{0}\right)+\int_{\Sigma_{\varepsilon}}\left(\beta^{\varepsilon}(x)-\beta^{0}\right) d x,
\end{aligned}
$$

where $E_{0}$ denotes the minimizer of $\mathscr{J}_{0}$. Also, we have

$$
\mathscr{J}_{\varepsilon}\left(E_{0}\right)=\mathscr{J}_{0}\left(E_{0}\right)+\int_{\Sigma_{0}}\left(\beta^{0}-\beta^{\varepsilon}(x)\right) d x
$$

Therefore,

$$
\mathscr{J}_{0}\left(E_{\varepsilon}\right) \leqslant \mathscr{J}_{0}\left(E_{0}\right)+\int_{\Sigma_{0}}\left(\beta^{0}-\beta^{\varepsilon}(x)\right) d x+\int_{\Sigma_{\varepsilon}}\left(\beta^{\varepsilon}(x)-\beta^{0}\right) d x .
$$

These two integrals are random variables. In the first integral, the domain of integration $\Sigma_{0}$ is fixed. In the second integral, the domain of integration $\Sigma_{\varepsilon}$ is random. However, Proposition 3.1 below shows that both integrals are small with high probability. Consequently, (3.4) and Proposition 3.1 imply

$$
\mathbb{P}\left(\mathscr{J}_{0}\left(E_{\varepsilon}\right) \geqslant \mathscr{J}_{0}\left(E_{0}\right)+\alpha\right) \leqslant C e^{-C \varepsilon^{-d} \alpha^{2}} .
$$

for all $\varepsilon \leqslant K_{r} \min \left(1, \alpha^{r}\right)$.

PROPOSITION 3.1 There are constants $K_{1}, K_{2}, K_{3}$ such that for all $\alpha>0$ and $\varepsilon \leqslant K_{1} \alpha$, we have

$$
\mathbb{P}\left(\left|\int_{\Sigma_{0}}\left(\beta^{\varepsilon}(x)-\beta^{0}\right) d x\right|>\alpha\right) \leqslant K_{2} e^{-K_{3} \varepsilon^{-d} \alpha^{2}} .
$$

If $r>(d+1) / d$, there is a constant $K_{r}$ such that for all $\alpha>0$ and $\varepsilon \leqslant K_{r} \min \left(1, \alpha^{r}\right)$,

$$
\left|\int_{\Sigma_{\varepsilon}}\left(\beta^{\varepsilon}(x)-\beta^{0}\right) d x\right| \leqslant \alpha
$$

also holds, except possibly on a set of measure $K_{2} e^{-K_{3} \varepsilon^{-d} \alpha^{2}}$.

Proof of Proposition 3.1. We first prove (3.5). Recall that $\beta^{\varepsilon}(x)=\beta_{k}^{\varepsilon}$ within the cube $Q_{k}^{\varepsilon}$. Let us define the set of indices $k \in \mathbb{Z}^{d}$ such that the cube $Q_{k}^{\epsilon}$ is contained within $\Sigma_{0}$ :

$$
\Lambda^{\varepsilon, 0}=\left\{k \in \mathbb{Z}^{d} ;\left|Q_{k}^{\varepsilon} \cap \Sigma_{0}\right|=\left|Q_{k}^{\varepsilon}\right|\right\} .
$$


We also define the set of indices

$$
\Gamma^{\varepsilon, 0}=\left\{k \in \mathbb{Z}^{d}:\left|Q_{k}^{\varepsilon} \cap \Sigma_{0}\right|>0,\left|Q_{k}^{\varepsilon} \cap\left(\mathbb{R}^{d} \backslash \Sigma_{0}\right)\right|>0\right\}
$$

corresponding to cubes overlapping the contact line $\partial \Sigma_{0}$. Therefore,

$$
\int_{\Sigma_{0}}\left(\beta^{\varepsilon}(x)-\beta^{0}\right) d x=\sum_{k \in \Lambda^{\varepsilon, 0}} \varepsilon^{d}\left(\beta_{k}^{\varepsilon}-\beta^{0}\right)+\sum_{k \in \Gamma^{\varepsilon, 0}} \int_{Q_{k}^{\varepsilon} \cap \Sigma_{0}}\left(\beta^{\varepsilon}(x)-\beta^{0}\right) d x .
$$

Because $\Sigma_{0}$ is a ball with positive radius $r=\sqrt{\rho^{2}-z_{0}^{2}}$, the number of $\varepsilon$-cubes intersecting the contact line $\partial \Sigma_{0}$ is bounded by $\left|\Gamma^{\varepsilon, 0}\right| \leqslant C V^{\frac{d-1}{d+1}} \varepsilon^{1-d}$. Therefore, since $\beta_{k}^{\varepsilon} \in(-1,1)$, the last term in (3.8) is bounded by

$$
\left|\sum_{k \in \Gamma^{\varepsilon, 0}} \int_{Q_{k}^{\varepsilon} \cap \Sigma_{0}}\left(\beta^{\varepsilon}(x)-\beta^{0}\right) d x\right| \leqslant \sum_{k \in \Gamma^{\varepsilon, 0}} 2\left|Q_{k}^{\varepsilon} \cap \Sigma_{0}\right| \leqslant 2\left|\Gamma^{\varepsilon, 0}\right| \varepsilon^{d} \leqslant C V^{\frac{d-1}{d+1}} \varepsilon
$$

with probability one.

We can estimate the first term in the right hand side of (3.8) using a concentration inequality for sums of independent random variables. Observe that the set of indices $\Lambda^{\varepsilon, 0}$ is deterministic, since $\Sigma_{0}$ is a deterministic set. Therefore, Hoeffding's inequality ( [12], Theorem 2) implies that

$$
\mathbb{P}\left(\left|\sum_{k \in \Lambda^{\varepsilon, 0}} \varepsilon^{d}\left(\beta_{k}^{\varepsilon}-\beta^{0}\right)\right|>\alpha\right) \leqslant 2 e^{-\frac{\varepsilon^{-2 d} \alpha^{2}}{2\left|\Lambda^{\varepsilon, 0}\right|}}
$$

holds for all $\alpha>0$ and $\varepsilon>0$, since $\beta_{k} \in(-1,1)$. We also have the bound $\left|\Lambda^{\varepsilon, 0}\right| \leqslant C V^{\frac{d}{d+1}} \varepsilon^{-d}$ and so

$$
\mathbb{P}\left(\left|\sum_{k \in \Lambda^{\varepsilon, 0}} \varepsilon^{d}\left(\beta_{k}^{\varepsilon}-\beta^{0}\right)\right|>\alpha\right) \leqslant 2 e^{-\frac{1}{2 C} \varepsilon^{-d} \alpha^{2} V^{-\frac{d}{d+1}}}
$$

By combining (3.9) and (3.11), we deduce that if $\varepsilon$ is such that

$$
C V^{\frac{d-1}{d+1}} \varepsilon \leqslant \frac{\alpha}{2}
$$

then

$$
\mathbb{P}\left(\left|\int_{\Sigma_{0}}\left(\beta^{\varepsilon}(x)-\beta^{0}\right) d x\right| \geqslant \alpha\right) \leqslant \mathbb{P}\left(\left|\sum_{k \in \Lambda^{\varepsilon, 0}} \varepsilon^{d}\left(\beta_{k}^{\varepsilon}-\beta^{0}\right)\right|>\frac{\alpha}{2}\right) \leqslant 2 e^{-C \varepsilon^{-d} \alpha^{2}},
$$

which is the estimate (3.5).

Now we prove (3.6). The main difficulty here is that the set $\Sigma_{\varepsilon}$ is a random set, so the argument used to prove (3.5) does not work unless we have some control on the regularity of the set $\Sigma_{\epsilon}$. For a large integer $N>1$ (to be chosen later) we set $h=N \varepsilon$ and define the family of open cubes $Q_{k}^{h} \subset \mathbb{R}^{d}$ by

$$
Q_{k}^{h}=h k+(0, h)^{d}, \quad k \in \mathbb{Z}^{d} .
$$


These cubes have volume $h^{d}=N^{d} \epsilon^{d}$. For $h>0$, let $\Lambda^{h, \varepsilon}$ be the set of all indices $k \in \mathbb{Z}^{d}$ such that the cube $Q_{k}^{h}$ is contained in $\Sigma_{\varepsilon}$ :

$$
\Lambda^{h, \varepsilon}=\left\{k \in \mathbb{Z}^{d} ;\left|Q_{k}^{h} \cap \Sigma_{\varepsilon}\right|=\left|Q_{k}^{h}\right|\right\} .
$$

Let $\Gamma^{h, \varepsilon}$ be the set of all indices $k$ such that cube $Q_{k}^{h}$ straddles the contact line $\partial \Sigma_{\varepsilon}$ :

$$
\Gamma^{h, \varepsilon}=\left\{k \in \mathbb{Z}^{d}:\left|Q_{k}^{h} \cap \Sigma_{\varepsilon}\right|>0,\left|Q_{k}^{h} \cap\left(\mathbb{R}^{d} \backslash \Sigma_{\varepsilon}\right)\right|>0\right\} .
$$

We will use $\left|\Lambda^{h, \varepsilon}\right|$ and $\left|\Gamma^{h, \varepsilon}\right|$ to denote the cardinality of these index sets. Now the integral in (3.6) may be decomposed as

$$
\int_{\Sigma_{\varepsilon}}\left(\beta^{\varepsilon}(x)-\beta^{0}\right) d x=\sum_{k \in \Lambda^{h, \varepsilon}} \int_{Q_{k}^{h}}\left(\beta^{\varepsilon}(x)-\beta^{0}\right) d x+\sum_{k \in \Gamma^{h, \varepsilon}} \int_{Q_{k}^{h} \cap \Sigma_{\varepsilon}}\left(\beta^{\varepsilon}(x)-\beta^{0}\right) d x
$$

The integrals in the last sum are over cubes $Q_{k}^{h}$ that intersect the random contact line $\partial \Sigma_{\varepsilon}$. Because of the regularity of the contact line, the number of disjoint cubes of size $h$ covering $\partial \Sigma_{\varepsilon}$ cannot be too large:

LEMmA 3.1 There is a universal constant $C$ such that $\left|\Gamma^{h, \epsilon}\right| \leqslant C V^{\frac{d-1}{d+1}} h^{1-d}$ holds for all $h, \epsilon>0$.

The proof of Lemma 3.1 is almost identical to that of Lemma 3.2 below, so we omit it. Therefore, since $\beta_{k}^{\varepsilon} \in(-1,1)$, the second sum in (3.13) is bounded by

$$
\left|\sum_{k \in \Gamma^{h, \varepsilon}} \int_{Q_{k}^{h} \cap \Sigma_{\varepsilon}}\left(\beta^{\varepsilon}(x)-\beta^{0}\right) d x\right| \leqslant \sum_{k \in \Gamma^{h, \varepsilon}} 2\left|Q_{k}^{h} \cap \Sigma_{\varepsilon}\right| \leqslant 2\left|\Gamma^{h, \varepsilon}\right| h^{d} \leqslant C V^{\frac{d-1}{d+1}} h
$$

with probability one. This is bounded by $\alpha / 2$ if $C V^{\frac{d-1}{d+1}} h \leqslant \alpha / 2$.

In the first sum appearing on the right hand side of (3.13), the integrals are over all cubes $Q_{k}^{h}$ that are contained within $\Sigma_{\varepsilon}$. The collection of such cubes is random. Nevertheless, it is not an arbitrary collection, since the boundary of their union cannot be too irregular, as we will show. Given a set of indices $S \subset \mathbb{Z}^{d}$, let $\partial S$ denote the set of indices $k \in S$ such that $k+v \in \mathbb{Z}^{d} \backslash S$ for some $v \in \mathbb{Z}^{d}$ with $|v|=1$. So, if we define

$$
D(S)=\bigcup_{k \in S} \overline{Q_{k}^{h}}
$$

then $\partial S$ is the collection of indices corresponding to cubes $Q_{k}^{h} \subset D(S)$ having a face on the boundary of $D(S)$. For $S=\Lambda^{h, \epsilon}$, we have the following estimate:

LEMma 3.2 There is a universal constant such that $\left|\partial \Lambda^{h, \epsilon}\right| \leqslant C V^{\frac{d-1}{d+1}} h^{1-d}$ holds for all $h, \epsilon>0$.

We will postpone the proof of this estimate and finish the proof of Proposition 3.1. Suppose $S \subset \mathbb{Z}^{d}$ is a fixed set of indices having cardinality $|S|=s^{d}$. Since $h=N \varepsilon$ each cube $Q_{k}^{h}$ contains $N^{d}$ cubes of size $\varepsilon$, and there are $(s N)^{d}$ cubes of size $\varepsilon$ contained in $\cup_{k \in S} Q_{k}^{h}$. Therefore, since the 
random variables $\beta^{j}$ are independent and identically distributed, we have

$$
\begin{aligned}
\mathbb{P}\left(\left|\sum_{k \in S} \int_{Q_{k}^{h}}\left(\beta^{\varepsilon}(x)-\beta^{0}\right) d x\right|>\alpha\right) & =\mathbb{P}\left(\left|\sum_{k \in S} \sum_{\substack{j \in \mathbb{Z}^{d} \\
Q_{j}^{\varepsilon} \subset Q_{k}^{h}}} \varepsilon^{d}\left(\beta^{j}-\beta^{0}\right)\right|>\alpha\right) \\
& =\mathbb{P}\left(\left|\sum_{j \in \mathbb{Z}^{d} \cap[0, N s)^{d}} \varepsilon^{d}\left(\beta^{j}-\beta^{0}\right)\right|>\alpha\right) .
\end{aligned}
$$

So, applying Hoeffding's inequality to (3.15), we conclude that

$$
\mathbb{P}\left(\left|\sum_{k \in S} \int_{Q_{k}^{h}}\left(\beta^{\varepsilon}(x)-\beta^{0}\right) d x\right|>\alpha\right) \leqslant 2 e^{-\frac{1}{2}(h \varepsilon)^{-d} s^{-d} \alpha^{2}}
$$

holds for the fixed set of indices $S$.

By definition of $\Lambda^{h, \epsilon}$ we know that $\left|\Lambda^{h, \varepsilon}\right| \leqslant h^{-d}\left|\Sigma_{\epsilon}\right| \leqslant C_{1} V^{(d / d+1)} h^{-d}$. Also, $\Sigma_{\varepsilon} \subset B_{R}(0)$, so $\Lambda^{h, \varepsilon} \subset B_{2 R / h}(0)$. These bounds and Lemma 3.2 show that, with probability one, the random index set $\Lambda^{h, \epsilon}$ must be an element of

$$
G_{h}=\left\{S \subset \mathbb{Z}^{d} \cap B_{2 R / h}(0)|| S\left|\leqslant C_{1} V^{(d / d+1)} h^{-d}, \quad\right| \partial S \mid \leqslant C V^{\frac{d-1}{d+1}} h^{1-d}\right\} .
$$

We refer to $G_{h}$ as the collection of admissible index sets, and we use $\left|G_{h}\right|$ to denote the cardinality of $G_{h}$ (i.e. the number of admissible sets). A key point in our analysis is that the number of admissible sets does not grow too fast as $h \rightarrow 0$ :

LEMmA 3.3 There is a universal constant $C$ such that $\log \left|G_{h}\right| \leqslant C h^{1-d}|\log (h)|$ for all $h \in$ $(0,1 / 2)$.

For $\alpha, h$, and $\varepsilon$ fixed and $S \in G_{h}$, let us define the $\mathcal{F}$-measurable set $\Theta_{S} \subset H$ by

$$
\Theta_{S}=\left\{\omega \in H:\left|\sum_{k \in S} \int_{Q_{k}^{h}}\left(\beta^{\varepsilon}(x)-\beta^{0}\right) d x\right|>\alpha\right\} .
$$

Since $\Lambda^{h, \varepsilon} \in G_{h}$, we observe that

$$
\left\{\omega \in H:\left|\sum_{k \in \Lambda^{h, \varepsilon}} \int_{Q_{k}^{h}}\left(\beta^{\varepsilon}(x)-\beta^{0}\right) d x\right|>\alpha\right\} \subset \bigcup_{S \in G_{h}} \Theta_{S} .
$$

Moreover, from (3.16) and the fact that $|S| \leqslant C_{1} V^{d /(d+1)} h^{-d}$ for all admissible index sets, we have

$$
\mathbb{P}\left(\bigcup_{S \in G_{h}} \Theta_{S}\right) \leqslant \sum_{S \in G_{h}} \mathbb{P}\left(\Theta_{S}\right) \leqslant\left|G_{h}\right| 2 \exp \left(-\frac{1}{2} \varepsilon^{-d} \alpha^{2} C_{1}^{-1} V^{-1 /(d+1)}\right)
$$


where $\left|G_{h}\right|$ is the cardinality of $G_{h}$, the number of admissible index sets. Therefore, by Lemma 3.3 we have

$$
\mathbb{P}\left(\bigcup_{S \in G_{h}} \Theta_{S}\right) \leqslant C_{3} \exp \left\{\varepsilon^{-d}\left(C_{2} \in N^{1-d} \log (h)-\frac{1}{2} \alpha^{2} C_{1}^{-1} V^{-1 /(d+1)}\right)\right\} .
$$

Considering both (3.14) and (3.19), we now choose $N=\left\lceil\epsilon^{-p}\right\rceil$ with suitable $p$ so that $C V^{\frac{d-1}{d+1}} h \leqslant$ $\alpha / 2$ holds and so that the exponent in (3.19) is negative for $\epsilon$ sufficiently small. Let us choose $p=1 /(d+1)$ and set $N=\left\lceil\epsilon^{-p}\right\rceil, h=\epsilon N=\epsilon^{1-p}=\epsilon^{d /(d+1)}$. Then $\epsilon N^{1-d} \log (h)=$ $(1-p) \epsilon^{1+p(d-1)} \log (\varepsilon) \leqslant(1-p) \epsilon^{2 d /(d+1)} \log (\varepsilon)$. Therefore, if $r>(d+1) / d$ there is a constant $C_{r}$ such that

$$
C_{2} \in N^{1-d} \log (h) \leqslant \frac{1}{4} \alpha^{2} C_{1}^{-1} V^{-1 /(d+1)}
$$

holds for all $\varepsilon \leqslant C_{r} \min \left(1, \alpha^{r}\right)$. The condition $C V^{\frac{d-1}{d+1}} h \leqslant \alpha / 2$ is also satisfied for such $r$. Therefore, (3.14) and (3.19) imply that

$$
\left|\int_{\Sigma_{\varepsilon}}\left(\beta^{\varepsilon}(x)-\beta^{0}\right) d x\right| \leqslant \frac{\alpha}{2}+\left|\sum_{k \in \Lambda^{h, \varepsilon}} \int_{Q_{k}^{h}}\left(\beta^{\varepsilon}(x)-\beta^{0}\right) d x\right| \leqslant \alpha,
$$

except possibly on the set $\cup_{S \in G_{h}} \Theta_{S}$ which has measure less than $C e^{-C \varepsilon^{-d} \alpha^{2}}$. This proves (3.6).

Proof of Lemma 3.2. This is similar to the proof of Proposition 2.3. If $k \in \partial \Lambda^{h, \epsilon}$, then $k \in \Lambda^{h, \varepsilon}$ and there exists $v \in \mathbb{Z}^{d}$ with $|v|=1$ such that $k+v \notin \Lambda^{h, \varepsilon}$. Therefore, there must be a point $x_{k} \in \overline{Q_{k+v}^{h}}$ for which $x_{k} \in \partial \Sigma_{\epsilon}$ (the contact line). By choosing such a point $x_{k}$ for each index $k \in \partial \Lambda^{h, \epsilon}$, we obtain a set of balls $B_{h / 2}^{+}\left(x_{k}, 0\right)$ with no more than $2^{d}$ of the balls overlapping. By Corollary 2.1 we know that $P\left(E_{\epsilon}, B_{h / 2}^{+}\left(x_{k}, 0\right)\right) \geqslant C h^{d}$. Therefore,

$$
\sum_{k \in \partial \Lambda^{h, \varepsilon}} P\left(E_{\epsilon}, B_{h / 2}^{+}\left(x_{k}, 0\right)\right) \leqslant C P\left(E_{\epsilon},\{0<z<h / 2\}\right) \leqslant C V^{\frac{d-1}{d+1}} h / 2 .
$$

Hence, the cardinality of $\partial \Lambda^{h, \varepsilon}$ is bounded by $\left|\partial \Lambda^{h, \varepsilon}\right| \leqslant C V^{\frac{d-1}{d+1}} h^{1-d}$.

Proof of Lemma 3.3. If $S \in G_{h}$, then $S \subset \mathbb{Z}^{d} \cap B_{2 R / h}(0)$, so there are at most $M=\left\lceil C h^{-d}\right\rceil$ cubes that can belong to $S$. Moreover, the set $S$ is uniquely determined by the cubes that have faces on the boundary of $D(S)$. That is, $S_{1}=S_{2}$ if and only if $\partial S_{1}=\partial S_{2}$. By definition of $G_{h}$, we know that $|\partial S| \leqslant C h^{1-d} \leqslant C M^{q}$ with $q=(d-1) / d$. Therefore, the number of possible sets in $G_{h}$ is bounded by

$$
\left|G_{h}\right| \leqslant \sum_{n=0}^{C M^{q}}\left(\begin{array}{c}
M \\
n
\end{array}\right) \leqslant(M+1)^{C M^{q}} .
$$

Therefore, we have $\log \left|G_{h}\right| \leqslant C h^{1-d} \log \left(C h^{-d}+1\right)$. 


\section{REFERENCES}

1. Caffarelli, L. \& Mellet, A., Capillary drops on an inhomogeneous surface. Perspectives in Nonlinear PDE. In honor of Haim Brezis. Contemp. Math. 446, Amer. Math. Soc., Providence, RI (2007), 175-201. Zb11200.76053 MR2373730

2. Caffarelli, L. \& Mellet, A. Capillary drops on an inhomogeneous surface: Contact angle hysteresis and sticking drop, Calc. Var. Partial Differential Equations 29 (2007), 141-160. Zbl1168.76007 MR2307770

3. CAFFARElli, L. \& Souganidis, P. E., Rates of convergence for the homogenization of fully nonlinear uniformly elliptic pde in random media, Inventiones Math. 108 (2010), 301-360. Zbl1192.35048 MR2609244

4. FInN, R., Equilibrium Capillary Surfaces. Springer-Verlag, New York (1986). Zb10583.35002 MR0816345

5. GiUsti, E., Minimal surfaces and functions of bounded variation. Monographs in Mathematics 80, Birkhäuser Verlag, Basel (1984). Zb10545. 49018 MR0775682

6. GonZalez, E., Sul problema della goccia appoggiata, Rend. Sem. Mat. Univ. Padova 55 (1976), 289-302. Zb10361.49027 MR0493670

7. Gonzalez, E., Massari, U. \& TAMAnini, I., On the regularity of boundaries of sets minimizing perimeter with a volume constraint. Indiana Univ. Math. J. 32 (1983), 25-37. Zbl0486 . 49024 MR0684753

8. KIM, I., Error estimate on homogenization of free boundary velocities, Ann. Inst. H. Poincaré Anal. Non Linéaire 26 (2009), 999-1019. Zbl1177 . 35260 MR2526413

9. KIM, I., Homogenization of free boundary velocities, Arch. Ration. Mech. Anal. 185 (2007), 69-103. Zbl1162.35011 MR2308859

10. KIM, I., Homogenization of a model problem on contact angle dynamics, Comm. PDE. 33 (2008), 12351271. Zb11161. 35312 MR2450158

11. Kim, I. \& Mellet, A., Homogenization of a Hele-Shaw problem in periodic and random media, Arch. Rat. Mech. Ana. 194 (2009), 507-530. Zblpre05640834 MR2563637

12. Hoeffining, W., Probability inequalities for sums of bounded random variables, J. Amer. Stat. Assoc. 58 (1963), 13-30. Zbl0127.10602 MR0144363 